This document is the accepted manuscript version of the following article:

Donnel1y, C., Metlov, K. L., Scagnoli, V., Guizar-Sicairos, M., Holler, M., Bingham, N. S., ... Gliga, S. (2020). Experimental observation of vortex rings in a bulk magnet. Nature Physics. https://doi.org/10.1038/s41567-020-01057-3

\title{
Experimental Observation of Vortex Rings in a Bulk Mag- net
}

Claire Donnelly ${ }^{1,2,3}$, Konstantin L. Metlov ${ }^{4,5}$, Valerio Scagnoli ${ }^{2,3}$, Manuel Guizar-Sicairos ${ }^{3}$, Mirko Holler $^{3}$, Nicholas S. Bingham ${ }^{2,3}$, Jörg Raabe ${ }^{3}$, Laura J. Heyderman ${ }^{2,3}$, Nigel R. Cooper ${ }^{1}$ and Sebastian Gliga ${ }^{3}$

${ }^{1}$ Cavendish Laboratory, University of Cambridge, JJ Thomson Ave, Cambridge CB3 OHE, UK.

${ }^{2}$ Laboratory for Mesoscopic Systems, Department of Materials, ETH Zürich, 8093 Zürich, Switzerland.

${ }^{3}$ Paul Scherrer Institute, 5232 Villigen PSI, Switzerland.

${ }^{4}$ Donetsk Institute for Physics and Engineering, R. Luxembourg 72, Donetsk 83114, Ukraine.

${ }^{5}$ Institute for Numerical Mathematics RAS, 8 Gubkina str., 119991 Moscow GSP-1, Russia.

Vortex rings are remarkably stable structures occurring in a large variety of systems: for example in turbulent gases, where they are at the origin of weather phenomena ${ }^{1}$; in fluids with implications for biology ${ }^{2}$; in electromagnetic discharges ${ }^{3}$; and in plasmas ${ }^{4}$. While vortex rings have also been predicted to exist in ferromagnets ${ }^{5}$, they have not yet been observed. Using X-ray magnetic nanotomography ${ }^{6}$, we imaged three-dimensional structures forming closed vortex loops in a bulk micromagnet. The cross-section of these loops consists of a vortex-antivortex pair and, based on magnetic vorticity, a quantity analogous to hydrodynamic vorticity, we identify these configurations as magnetic vortex rings. While such structures have been predicted to exist as transient states in exchange ferromagnets ${ }^{5}$, the 
vortex rings we observe exist as stable, static configurations, whose stability we attribute to the dipolar interaction. In addition, we observe stable vortex loops intersected by magnetic singularities $^{7}$, at which the magnetisation within the vortex and antivortex cores reverses. We gain insight into the stability of these states through field and thermal equilibration protocols. The observation of stable magnetic vortex rings opens possibilities for further studies of complex three-dimensional solitons in bulk magnets, enabling the development of applications based on three-dimensional magnetic structures.

In magnetic thin films, vortices are naturally occurring flux closure states, in which the magnetisation curls around a stable core, where the magnetisation tilts out of the film plane ${ }^{8,9}$. These structures have been studied extensively over the past decades due to their intrinsic stability ${ }^{10}$ and their topology-driven dynamics ${ }^{11-13}$, which are of both fundamental and technological ${ }^{14}$ interest. Antivortices, the topological counterpart of vortices, distinguish themselves from vortices by an opposite rotation of the in-plane magnetization that is quantified by the index of the vector field which is equal to the winding number of a path traced by the magnetisation vector while moving in the counterclockwise direction around the core ${ }^{15}$. While vortices have a circular symmetry of the magnetisation (figure 1a), antivortices only display inversion symmetry about the center ${ }^{16}$ (figure 1b), resembling saddle points in the vector field. Experimental studies of magnetic vortices and antivortices have mostly been restricted to two dimensional, planar systems, in which vortex-antivortex pairs have a natural tendency to annihilate ${ }^{17}$, unless they are part of larger, stable structures, such as cross-tie walls ${ }^{18}$. 
In bulk ferromagnets, the existence of transient vortex rings, that take the form of localised solitons and are analogous to smoke rings, has been predicted ${ }^{5}$, but such structures have so far not been observed. Just as vortex rings in fluids are characterised by their vorticity, ferromagnetic vortex ring structures can be identified by considering the magnetic vorticity ${ }^{19}$. By analogy with fluid vorticity, the magnetic vorticity is a vector field, which can be defined as ${ }^{5,19}$ :

$$
\Omega_{\alpha}=\frac{1}{8 \pi} \epsilon_{\alpha \beta \gamma} \epsilon_{i j k} m_{i} \partial_{\beta} m_{j} \partial_{\gamma} m_{k}
$$

where $m_{i}(\mathbf{r}, t)$ is a component of the unit vector representing the local orientation of the magnetisation $\boldsymbol{m}=|\boldsymbol{M}| / M_{s}$, the reduced magnetisation, where $M_{s}$ is the saturation magnetisation, $\alpha$ indicates the vorticity component, and $\epsilon_{\alpha \beta \gamma}$ is the Levi-Civita tensor, summed over three components $x, y, z$. The magnetic vorticity vector $\Omega$ represents the topological charge flux ${ }^{20}$ (or Skyrmion number ${ }^{21}$ ) density. Integrating the magnetic vorticity over a closed two-dimensional surface $S$, results in an integer value $\int_{S} \Omega \cdot \mathrm{d} S=N$ corresponding to the Skyrmion number, which gives the degree of mapping of the magnetization distribution to an order parameter space described by the surface of an $S^{2}$ sphere. When $N=1$, the target sphere is wrapped exactly once and each direction of the magnetisation vector is present on the surface $S$. The magnetic vorticity vector $\Omega$ is therefore non-vanishing in the vicinity of the cores of vortices or antivortices, and is represented in Figure 1a-d for vortices and antivortices with different polarisations (the polarisation is the orientation of the magnetisation within the core). The vorticity vector is aligned parallel to the polarisation of a vortex $(a, c)$ and antiparallel to the polarisation of an antivortex $(b, d)$, indicating that it is dependent upon the direction of the magnetisation in the core as well as the index of the structure. Consequently, a vortex-antivortex pair with parallel polarisations exhibit opposite 
vorticities, that circulate in a closed loop (Figure 1e).

Here, we use the magnetic vorticity to locate and identify magnetization structures within a three-dimensional $\mathrm{GdCo}_{2}$ micropillar, imaged using hard X-ray magnetic nanotomography ${ }^{6}$. Within the bulk of the pillar, we find two types of vorticity loops. The first is characterised by a circulating magnetic vorticity forming vortex rings, analogous to smoke rings. The cross-sections of these magnetic vortex rings consist of vortex-antivortex pairs with parallel polarisations, as illustrated in Figure 1e. Consequently, such a pair can be smoothly transformed into a uniformly magnetised state and carries zero topological charge. The second type of loop contains singularities, or Bloch points ${ }^{7}$, at which the vorticity abruptly reverses its sign, reflecting the reversal of the polarisation of the vortex and antivortex within the cross-section of the ring. Calculating preimages of the observed structures reveals concentric pre-images that do not link each other, so have a vanishing Hopf index (a topological invariant which counts the linking number of pre-images corresponding to different magnetization vector directions). In contrast, structures containing Bloch points have preimages similar to recently observed 'toron' structures in anisotropic fluids ${ }^{22}$.

The hard X-ray magnetic nanotomography setup is illustrated in Figure 1f. During the measurement, high resolution X-ray projections of a bulk $\mathrm{GdCo}_{2}$ ferrimagnetic cylinder of diameter 5 $\mu \mathrm{m}$ were measured with dichroic ptychography ${ }^{23}$ for 1024 orientations of the sample with respect to the X-ray beam. The photon energy of the circularly-polarised X-rays was tuned to the $\mathrm{Gd} L_{3}$ edge and, by exploiting the X-ray magnetic circular dichroism effect, sensitivity to the component of the magnetisation parallel to the X-ray beam was obtained. In order to gain access to all three 
components of the magnetisation, X-ray projections were measured for different sample orientations about the tomographic rotation axis for two different sample tilts. The internal magnetic structure was obtained using an iterative reconstruction algorithm ${ }^{6}$, which has been demonstrated to offer a robust reconstruction of nanoscale magnetic textures ${ }^{24}$. Further experimental details are given in the Methods section.

In the ferrimagnetic micropillar, the coupling between two antiparallel magnetic sublattices leads to an effective soft ferromagnetic behavior ${ }^{25}$. The lowest energy state of such a magnetic cylinder is expected to consist of a single vorte ${ }^{26}$. In practice, the size of the pillar is large enough to reduce the role of surface anisotropy, supporting the stabilisation of more complex, often metastable states, that can include a large number of vortices, anti-vortices, domain walls and singularities ${ }^{6}$.

We compute the magnetic vorticity $\Omega$ from the reconstructed magnetisation following equation (1). Regions of large vorticity are plotted in Figure 1g, where a number of 'tubes' and loops corresponding to the cores of vortices and antivortices are visible. In addition, unlike in incompressible fluids where the divergence must vanish, a non-zero divergence of the magnetisation, $\mathbf{M}$, is allowed in ferromagnets, given that Maxwell's equations only exclude the divergence of $\mathbf{B}$. In this way, computing the magnetic vorticity also allows us to locate singularities of the magnetisation - known as Bloch points - within the system, which are characterised by a large divergence of the magnetic vorticity, $\nabla \cdot \Omega$, due to the abrupt local variation in the orientation of the magnetisation. Here, Bloch points and anti-Bloch points are identified by positive (red) and negative (blue) 

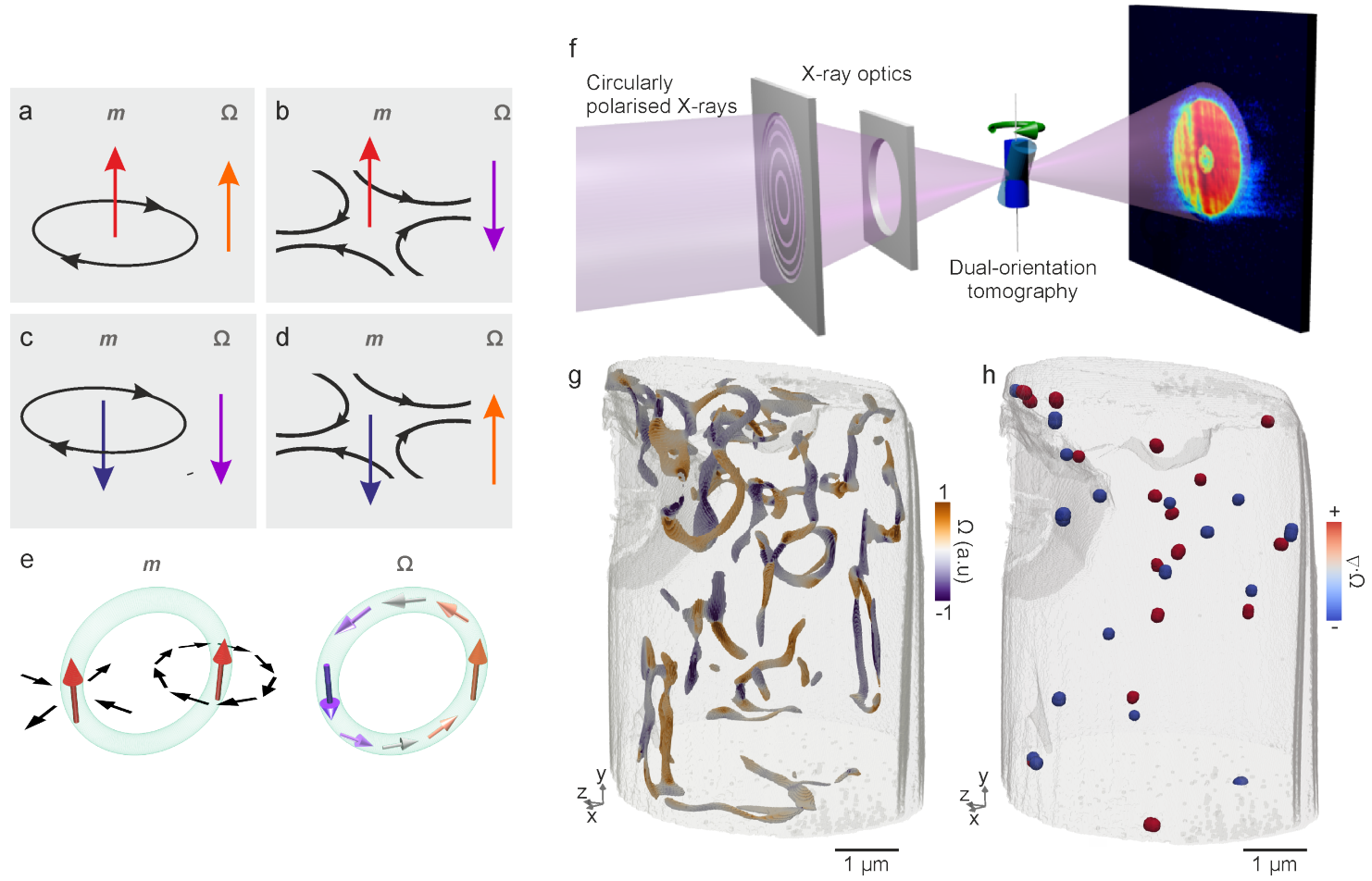

Figure 1: Measuring and reconstructing the magnetic structure and the magnetic vorticity within a $\mathrm{GdCo}_{2}$ pillar. a-d) Schematic representation of the magnetic vorticity $\Omega$, shown in purple and orange arrows, for a number of vortex $(\mathrm{a}, \mathrm{c})$ and antivortex $(\mathrm{b}, \mathrm{d})$ configurations with different polarisations (red, dark blue). The vorticity of a ring composed of a vortex-antivortex pair with parallel polarisations is shown in (e). f) Schematic representation of the experimental setup: tomographic projections with magnetic contrast are measured using dichroic ptychography for the sample at many different azimuthal angles with respect to the X-ray beam (rotation indicated by green arrow). Measurements were performed with the sample at two different tilt angles: $30^{\circ}$ (transparent green cylinder) and $0^{\circ}$ (blue cylinder). g) Plotting regions of significant magnetic vorticity, we locate a variety of structures, and h) plotting regions of high divergence of the vorticity $\nabla \cdot \Omega$, we locate Bloch points (red) and anti-Bloch points (blue), which respectively have positive and negative $\nabla \cdot \Omega$. 
$\nabla \cdot \Omega$, as plotted in Figure $1 \mathrm{~h}$. Within the pillar, we find an equal number of Bloch points and antiBloch points, indicating that the singularities most likely originated in the bulk of the structure, where they can only be created in pairs. As a result, it appears that sample boundaries, through which a single Bloch point could be injected, did not play an essential role in the formation of the observed structures.

Within the reconstructed magnetisation, we observe a large number of three-dimensional loops (Figure 2c), that resemble the vortex ring schematically illustrated in Figure 1e. We consider the case of one such loop, identified by plotting an isosurface corresponding to $\boldsymbol{m}= \pm \hat{\mathrm{x}}$ in Figure 2a. This loop is located in the vicinity of a single vortex extending throughout the majority of the height of the pillar and whose polarisation equally points along the $+\hat{\mathrm{x}}$ direction in the shown slice. Considering the magnetisation in the $y-z$ plane, represented by streamlines in Figure 2a, we identify a bound state consisting of two vortices separated by an antivortex, a structure analogous to that of a cross-tie wall. Note that the streamlines are used to indicate the direction of the magnetization and are extrapolated beyond the spatial resolution of the measurements. Similarly, the isosurfaces highlight the position of the vortex core and do not represent the width of the core. The loop itself is embedded within a quasi-uniformly magnetised region $(\mathbf{m}=+\hat{\mathbf{x}}$, red $)$ and therefore the vortex and antivortex have parallel polarisations, as shown schematically in Figure 1e. Calculating the magnetic vorticity vector $\Omega$, plotted in Figure $2 b$, reveals a unidirectional circulation around the loop, directly comparable to the schematic in Figure 1e. This structure is similar to a vortex ring in a fluid, which equally corresponds to a loop in the hydrodynamic vorticity. Such vorticity loops have been predicted to exist as propagating solitons in exchange ferromagnets ${ }^{5}$. In 

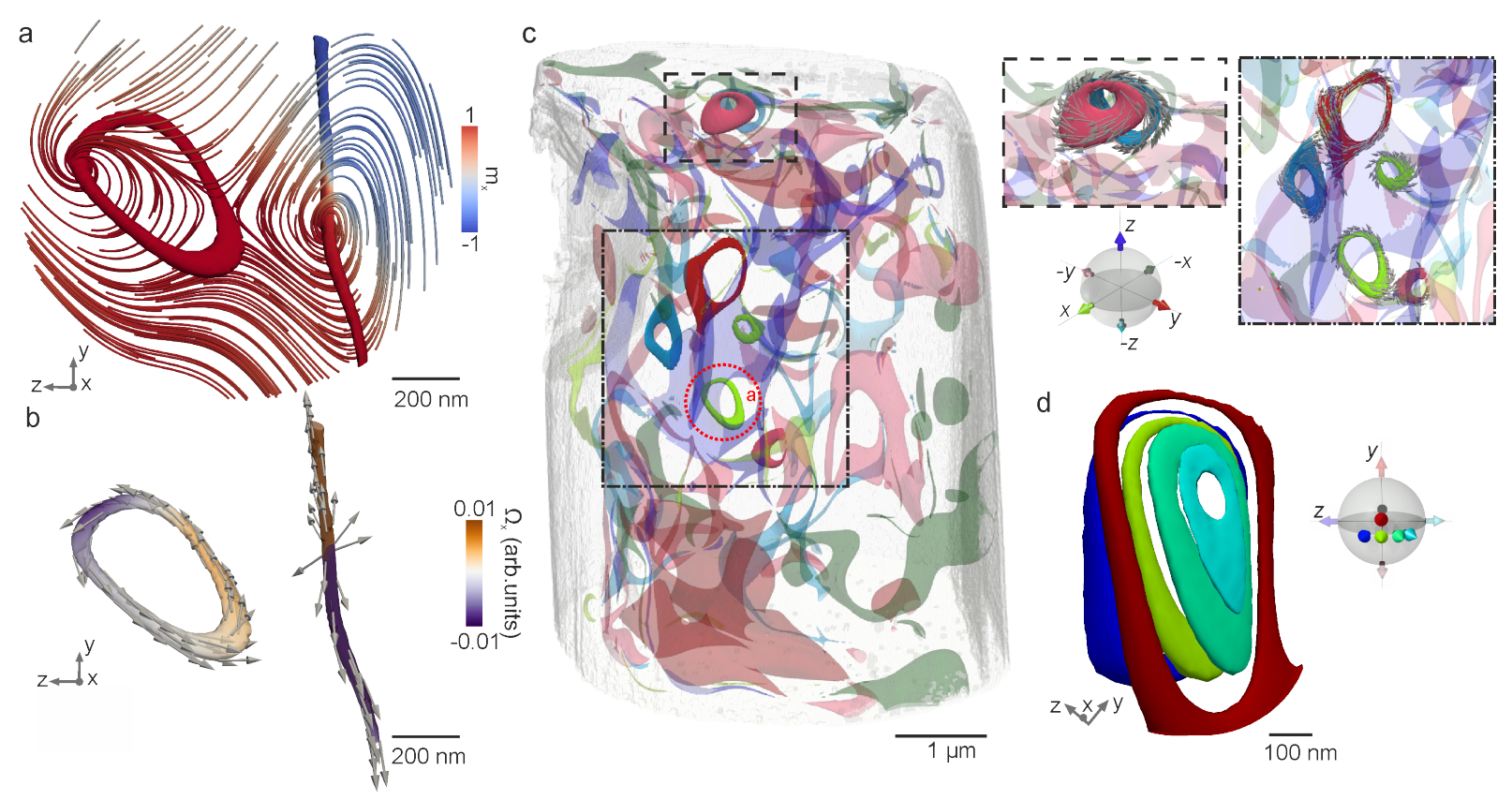

Figure 2: Structure of a vortex ring with circulating magnetic vorticity. a) A loop is identified next to a vortex by plotting an isosurface corresponding to $m_{x}= \pm 1$. The in-plane magnetisation within a two-dimensional slice through the loop is plotted using streamlines, revealing that the cross-section of the loop consists of a vortex-antivortex pair. The colourmap indicates the value of $m_{x}$, reevaling that the vortex and the antivortex within the loop have the same polarisation. b) On the same $m_{x}= \pm 1$ isosurface, mapping the vorticity (represented both by the arrows and the colourmap) reveals that the loop exhibits a circulating vorticity and is a vortex ring. The vorticity map equally indicates that, in the nearby extended vortex, the vorticity abruptly reverses, indicating the presence of a Bloch point. Note that the plotted structures have a relatively low vorticity, with $|\Omega| \simeq 0.1$ (with the exception of the Bloch point). c) Plotting preimages for different directions, indicated on the schematic sphere, reveals a number of closed loops within the sample. Calculating the vorticity reveals that these loops also correspond to vortex rings (insets). d) In the vicinity of the vortex loop in a), preimages for ngighbouring directions are not linked, indicating a Hopf index of zero. 
contrast, the vortex loops observed here are static and stable at room temperature over the duration of our measurements. We note that the diameter of the vortex ring, i.e. the average distance between the vortex and antivortex cores in the $y-z$ plane, is approximately $370 \mathrm{~nm}$, and is comparable to the diameter of other vortex rings present inside the pillar (see Figure 2c) that exhibit an average diameter of $400 \pm 90 \mathrm{~nm}$. Interestingly, this loop (along with a number of similar vortex rings in the sample) occurs in the vicinity of a singularity: indeed, the neighbouring vortex in the cross-tie structure contains a Bloch point, which is located in Figure 2b where the vorticity, (and the magnetisation in the vortex core) abruptly reverses direction, as seen in Extended Data Figure 5. There is a priori no topological requirement for the presence of a Bloch point in proximity of the vortex loop and despite the observed correlations, our static observations do not allow for the determination of a causal relationship between the presence of both structures.

We gain further insight into the topology of these vortex loops by plotting preimages corresponding to a number of directions of the magnetisation in the vicinity of the vortex ring. The preimage corresponding to the $+\hat{\mathbf{x}}$ direction, i.e. $m_{x}=+1$, is plotted in light green in Figure $2 \mathrm{~d}$, along with additional preimages corresponding to directions indicated in the inset that form an ensemble of closed-loop preimages. The plotted loops do not link, indicating that the vortex ring has a Hopf number $H=0$. Indeed, the vicinity of the $H=0$ structure contains only preimages representing directions close to the $+\hat{\mathbf{x}}$ direction and, consequently, do not cover the $S^{2}$ sphere (as illustrated on the schematic sphere in Figure 2d), meaning that the magnetisation can smoothly unwind into a single point on the sphere ${ }^{27}$. Hence, these vortex rings belong to a class of nontopological solitons ${ }^{28}$. In the Methods (Extended Data Figure 3c), we have developed an analytic 
model of such a soliton, qualitatively reproducing the observed features, vorticity and pre-images.

In addition to vortex rings, we also identify loops containing sources and sinks of the magnetisation, due to the presence of Bloch points. The magnetic structure of one such loop, highlighted by the isosurface $m_{x}= \pm \hat{x}$, is shown in Figure 3a using streamlines, where the colourscale represents $m_{x}$ and the magnetisation in a plane of the loop is represented by streamlines, revealing a vortex-antivortex pair. At two points within the loop, the polarisation along the vortex and antivortex cores reverses (colour changes from blue to red). Consequently, the vorticity does not circulate around the loop, but instead assumes an asymmetric onion-like structure, flowing out from a source (green box in Figure $3 b$ ) and into a sink (orange box in Figure 3b). The structure of the magnetisation in the vicinity of the singularities is plotted in Figures $3 \mathrm{c}, \mathrm{d}$. The vorticity sink (Figure 3e), whose surrounding magnetisation is plotted in Figure 3c, corresponds to a contracirculating Bloch point ${ }^{29}$ (or anti-Bloch point) with Skyrmion number -1 . The vorticity source (Figure 3f), has a magnetisation structure (Figure 3d) corresponding to that of a circulating Bloch point ${ }^{29}$ with Skyrmion number +1 . Two features of this loop are particularly noteworthy. First, the singularities are not linked to the generation and annihilation of a vortex and antivortex with opposite polarisations, as has been reported for dynamic processes ${ }^{15}$. Instead, the loop consists of two halves connected by the Bloch points, which locally leads to a reversal of the vorticity along the vortex and the antivortex cores, as seen in Extended Data Figure 4. Second, while singularities often mediate dynamic magnetisation processes and have been predicted during magnetisation dynamics $^{29,30}$ as well as during magnetic field reconnection in plasma physics ${ }^{31}$, the observed structures are inherently static. In Ref. 6, Bloch points were observed at the locations where a 

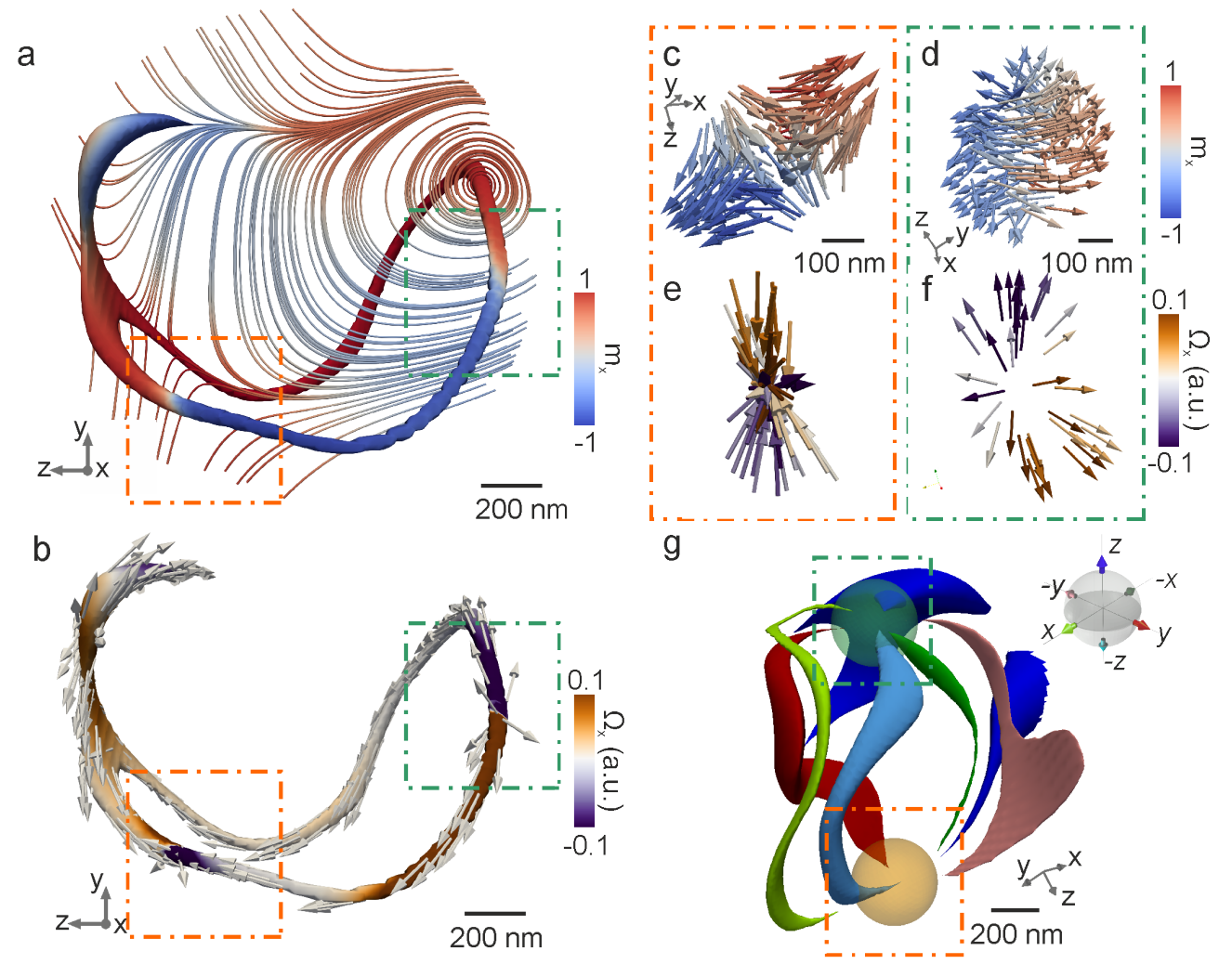

Figure 3: Structure of a vortex loop containing magnetization singularities. a) The loop is highlighted by the $m_{x}= \pm \hat{x}$ isosurface, while the magnetic configuration in a two-dimensional slice is plotted using streamlines, with the colour indicating the out-of-plane magnetisation component $\pm m_{x}$. The cross-section contains a vortex-antivortex pair. Within the loop, the polarisations of the vortex and anti-vortex cores switch from $+m_{x}$ (red) to $-m_{x}$ (blue) at two points, indicated by the orange and green boxes. b) The magnetic vorticity forms an "onion" state, with the vorticity direction reversing at the same two points. These locations correspond to singularities of the magnetisation, whose surrounding magnetic and vorticity structure is plotted in (c,d) and (e,f), respectively. g) Preimages corresponding to the Cartesian axes $\pm \hat{\mathbf{x}}$ (light/dark green), $\pm \hat{\mathbf{y}}$ (light/dark red), and $\pm \hat{\mathbf{z}}$ (light/dark blue) (indicated on the schematic sphere), which reveal an onion-like state, with all preimages meeting at the singularities. See also Extended Data Figure 7. 
vortex core intersected a domain wall. Similarly, we find that the Bloch point pair is located at the intersection of the vortex-antivortex loop with a domain wall separating regions of opposite $m_{x}$ (Extended Data Figure 5f).

We gain further insight into the topology of the vortex-antivortex loop containing singularities by plotting preimages corresponding to a defined set of spatial directions, (or points on the $S^{2}$ sphere) in Figure 3g. In particular, we plot regions of the magnetisation aligned along $\pm \hat{\mathbf{x}}$ (bright/ dark green), $\pm \hat{\mathbf{y}}$ (bright/ dark red), and $\pm \hat{\mathbf{z}}$ (bright/ dark blue), which form a three-dimensional onion state, with all directions of the magnetisation meeting at the singularities schematically indicated by green (Bloch point) and orange (anti-Bloch point) circles. The preimages resemble those found to correspond to 'torons', which have recently been observed in chiral liquid crystals ${ }^{32}$ and anisotropic fluids ${ }^{33}$. In the Methods, we present an analytical model describing different micromagnetic configurations with similar pre-images, allowing us to reproduce and, consequently, understand the experimental observations.

We explore the stability of the observed vorticity loops by applying two different field and thermal protocols on a similar $\mathrm{GdCO}_{2}$ micropillar, and performing magnetic X-ray nanotomography at remanence following each protocol. In the first protocol, we apply a $7 \mathrm{~T}$ magnetic field along the long axis of the pillar at room temperature, and image the resulting remanent configuration. The applied field is above the measured sample saturation field of $\sim 2 \mathrm{~T}$. A plot of the magnetic vorticity (Figure $4 \mathrm{a}$ ) reveals a large number of vortices and antivortices, as well as magnetic singularities (shown in Methods and Extended Data Figure 6 at remanence). Plotting 
a

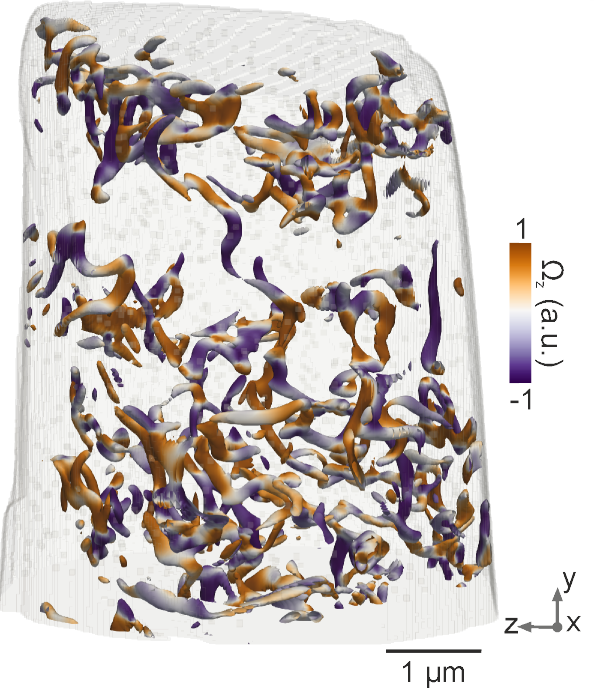

b
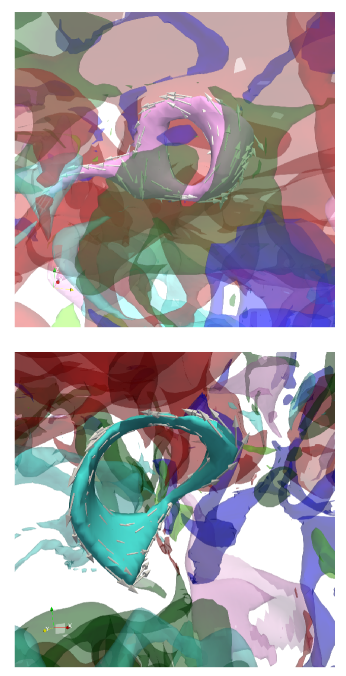

C

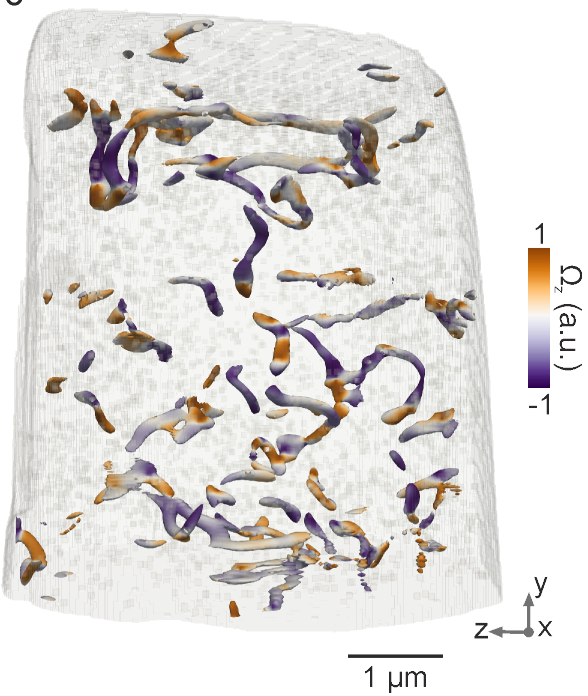

Figure 4: Magnetic vorticity plots measured for for a similar micropillar at remanence showing the effect of different field histories. a) Following the application of a $7 \mathrm{~T}$ saturating field, a small number of vortex rings like the one plotted in Figure 2 are present at remanence, some of which are shown in b). c) After annealing in a $7 \mathrm{~T}$ field, followed by field cooling, no rings are observed. 
preimages corresponding to different directions of the magnetisation, we observe a small number of vortex loops, two of which are shown in figure $4 \mathrm{~b}$. The presence of these vortex loops after the application of a saturating magnetic field indicates that the loops can nucleate spontaneously, and therefore do not require a specific field protocol to prepare them. Secondly, we heat the sample to $400 \mathrm{~K}$ while applying a $7 \mathrm{~T}$ magnetic field. The sample is then field cooled and the field gradually removed after the sample reaches room temperature. This annealing procedure is reminiscent of those used to expel defects in single-crystals in order to increase their purity. A plot of the vorticity, shown in figure 4c, reveals a noticeably smaller number of structures with non-vanishing vorticity. Importantly, we do not find any vortex loops, indicating that these are metastable states that are more efficiently destroyed through thermal annealing in a field, which is likely to lead to the expulsion of magnetic as well as lattice defects that contribute to pinning of the magnetic structures (see Methods and Extended Data Figures 1 and 2 for more details). Quantitatively, the average vorticity value following field cooling is half the value following only the application of a $7 \mathrm{~T}$ field, and the total number of Bloch points is roughly halved (52 vs. 110 Bloch points, as seen in Extended Data Figure 6).

Although the vortex rings we observe are topologically trivial structures and have a Hopf index of zero, they are surprisingly stable. We attribute their stability to interactions with surrounding magnetization structures, which ensure that they are, for example, embedded in cross-tie structures. In the case of the loops containing Bloch points, the singularities occur at the intersection with domain walls (as shown in Extended Data Figure 6), thus pinning the loops. Moreover, the magnetostatic interaction clearly plays an important role in the stabilisation of these structures, 
ensuring that our observations of stable localised solitons do not contradict the Hobart-Derrick theorem for an exchange ferromagnet that requires non-linearities (such as intrinsic chirality in the presence of Dzyaloshinskii-Moriya interaction) to set a scale for localised magnetisation nonuniformities. Based on the balance of magnetostatic and exchange interactions, a distance of $\approx 296 \mathrm{~nm}$ between the vortex and antivortex in such bound states can be estimated via the bulk limit of the cross-tie domain wall width as described in the Methods section. This value matches the average observed size of the rings of $400 \pm 90 \mathrm{~nm}$, indicating that the balance of the magnetostatic and exchange interactions is sufficient to stabilise the structures. Details of the model are given in the Methods. We note that chirality has been demonstrated in a similar bulk amorphous system through the inclusion of structural inhomogeneities ${ }^{34}$. We expect that such systems could host topologically non-trivial solitons, such as knots with a higher Hopf number, as well as torons, following predictions for chiral magnetic heterostructures ${ }^{33,35,36}$, analogous to the reported observations in chiral liquid crystals and ferrofluids ${ }^{27,37}$.

The calculation and visualization of the magnetic vorticity and of preimages have proven essential tools in the characterisation of the observed three-dimensional structures. In combination with recent advances in time-resolved X-ray magnetic laminography ${ }^{38}$, these open the path to investigating the dynamics of three-dimensional magnetic solitons. As well as probing resonant dynamics, it is possible that investigations of the displacement of three-dimensional vortex rings could reveal behaviour analogous to the Kelvin motion of two-dimensional vortex-antivortex pairs 39-41. Likewise, we expect that the magnetic vortex loops discovered here containing singularities will also display compelling dynamics, with implications for the fundamental understanding of the 
role of singularities in magnetisation processes. Finally, the study of the conditions for the formation of three-dimensional magnetic structures, and of their stability, is expected to lead to new possibilities for the controlled manipulation of the magnetisation that could be relevant for technological applications requiring complexity, such as neuromorphic computing ${ }^{42}$ or new proposals for three-dimensional data storage ${ }^{43}$.

1. Yao, J. \& Lundgren, T. Experimental investigation of microbursts. Experiments in Fluids 21, $17-25$ (1996).

2. Kilner, P. J. et al. Asymmetric redirection of flow through the heart. Nature 404, 759 - 761 (2000). Article.

3. Stenhoff, M. Ball Lightning: An Unsolved Problem in Atmospheric Physics (Springer-Verlag, US, 1999), 1 edn.

4. Akhmetov, D. G. Vortex Rings (Springer-Verlag, Berlin, Heidelberg, 2009), 1 edn.

5. Cooper, N. R. Propagating Magnetic Vortex Rings in Ferromagnets. Phys. Rev. Lett. 82, 1554-1557 (1999).

6. Donnelly, C. et al. Three-dimensional magnetization structures revealed with X-ray vector nanotomography. Nature 547, 328-331 (2017).

7. Feldtkeller, E. Mikromagnetisch stetige und unstetige magnetisierungskonfigurationen. Zeitschrift für angewandte Physik 19, 530-536 (1965). 
8. Shinjo, T., Okuno, T., Hassdorf, R., Shigeto, K. \& Ono, T. Magnetic Vortex Core Observation in Circular Dots of Permalloy. Science 289, 930-932 (2000).

9. Wachowiak, A. et al. Direct observation of internal spin structure of magnetic vortex cores. Science 298, 577-580 (2002).

10. Guslienko, K. Magnetic vortex state stability reversal and dynamics in restricted geometries. Journal of nanoscience and nanotechnology 8, 2745-60 (2008).

11. Choe, S.-B. et al. Vortex core-driven magnetization dynamics. Science 304, 420-422 (2004). URL https://science.sciencemag.org/content/304/5669/420. https://science.sciencemag.org/content/304/5669/420.full.pdf.

12. Van Waeyenberge, B. et al. Magnetic vortex core reversal by excitation with short bursts of an alternating field. Nature 444, 461 - 464 (2006). Article.

13. Hertel, R., Gliga, S., Fähnle, M. \& Schneider, C. M. Ultrafast Nanomagnetic Toggle Switching of Vortex Cores. Phys. Rev. Lett. 98, 117201 (2007).

14. Pigeau, B. et al. A frequency-controlled magnetic vortex memory. Applied Physics Letters 96, 132506 (2010). URL https://doi.org/10.1063/1.3373833. https://doi.org/10.1063/1.3373833.

15. Hertel, R. \& Schneider, C. M. Exchange Explosions: Magnetization Dynamics during VortexAntivortex Annihilation. Phys. Rev. Lett. 97, 177202 (2006). 
16. Gliga, S., Yan, M., Hertel, R. \& Schneider, C. M. Ultrafast dynamics of a magnetic antivortex: Micromagnetic simulations. Phys. Rev. B 77, 060404 (2008).

17. Gliga, S., Hertel, R. \& Schneider, C. M. Switching a magnetic antivortex core with ultrashort field pulses. Journal of Applied Physics 103, 07B115 (2008).

18. Neudert, A. et al. Bloch-line generation in cross-tie walls by fast magnetic-field pulses. Journal of Applied Physics 99, 08F302 (2006). URL https://doi.org/10.1063/1.2170399. https://doi.org/10.1063/1.2170399.

19. Papanicolaou, N. Dynamics of Magnetic Vortex Rings, vol. 404 of Singularities in Fluids, Plasmas and Optics (NATO ASI Series C404, 1993).

20. Belavin, A. A. \& Polyakov, A. M. Metastable states of two-dimensional isotropic ferromagnet. ZETP lett. 22, 245-247 (1975).

21. Senthil, T., Vishwanath, A., Balents, L., Sachdev, S. \& Fisher, M. P. A. Deconfined quantum critical points. Science 303, 1490-1494 (2004). URL https://science.sciencemag.org/content/303/5663/1490. https://science.sciencemag.org/content/303/5663/1490.full.pdf.

22. Ackerman, P. J. \& Smalyukh, I. I. Diversity of Knot Solitons in Liquid Crystals Manifested by Linking of Preimages in Torons and Hopfions. Phys. Rev. X 7, 011006 (2017).

23. Donnelly, C. et al. High-resolution hard x-ray magnetic imaging with dichroic ptychography. Phys. Rev. B 94, 064421 (2016). 
24. Donnelly, C. et al. Tomographic reconstruction of a three-dimensional magnetization vector field. New Journal of Physics 20, 083009 (2018).

25. Chikazumi, S. Physics of ferromagnetism, vol. 94 of International Series of Monographs on Physics (Oxford University Press, Oxford ; New York, 2010), 2 edn.

26. Arrott, A., Heinrich, B. \& Aharoni, A. Point singularities and magnetization reversal in ideally soft ferromagnetic cylinders. IEEE Transactions on Magnetics 15, 1228-1235 (1979).

27. Ackerman, P. J. \& Smalyukh, I. I. Static three-dimensional topological solitons in fluid chiral ferromagnets and colloids. Nature Materials 16, 426-432 (2016).

28. Lee, T. \& Pang, Y. Nontopological solitons. Physics Reports 221, 251 - 350 (1992). URL http://www.sciencedirect.com/science/article/pii/0370157392900647.

29. Malozemoff, A. \& Slonczewski, J. IV - domain-wall statics. $\quad$ In Malozemoff, A. \& Slonczewski, J. (eds.) Magnetic Domain Walls in Bubble Materials, 77 - 121 (Academic Press, 1979). URL http://www.sciencedirect.com/science/article/pii/B978012002951850008X.

30. Miltat, J. \& Thiaville, A. Vortex cores-smaller than small. Science 298, 555-555 (2002). URL https://science.sciencemag.org/content/298/5593/555. https://science.sciencemag.org/content/298/5593/555.full.pdf.

31. Kerr, R. M. \& Brandenburg, A. Evidence for a singularity in ideal magnetohydrodynamics: Implications for fast reconnection. Phys. Rev. Lett. 83, 1155-1158 (1999). URL https://link.aps.org/doi/10.1103/PhysRevLett.83.1155. 
32. Smalyukh, I. I., Lansac, Y., Clark, N. A. \& Trivedi, R. P. Three-dimensional structure and multistable optical switching of triple-twisted particle-like excitations in anisotropic fluids. Nature Materials 9, 139-145 (2009). Article.

33. Liu, Y., Lake, R. K. \& Zang, J. Binding a hopfion in a chiral magnet nanodisk. Phys. Rev. B 98, 174437 (2018). URL https://link.aps.org/doi/10.1103/PhysRevB.98.174437.

34. Kim, D.-H. et al. Bulk DzyaloshinskiiMoriya interaction in amorphous ferrimagnetic alloys. Nat. Mater. 18, 685 - 690 (2019). Article.

35. Sutcliffe, P. Hopfions in chiral magnets. Journal of Physics A: Mathematical and Theoretical 51, 375401 (2018). URL https: / / doi .org/10 .1088/1751-8121/aad521.

36. Tai, J.-S. B. \& Smalyukh, I. I. Static hopf solitons and knotted emergent fields in solid-state noncentrosymmetric magnetic nanostructures. Phys. Rev. Lett. 121, 187201 (2018). URL https://link.aps.org/doi/10.1103/PhysRevLett.121.187201.

37. Chen, B. G.-g., Ackerman, P. J., Alexander, G. P., Kamien, R. D. \& Smalyukh, I. I. Generating the Hopf Fibration Experimentally in Nematic Liquid Crystals. Phys. Rev. Lett. 110, 237801 (2013).

38. Donnelly, C. et al. Time-resolved imaging of three-dimensional nanoscale magnetisation dynamics. Nature Nanotechnology 15, 356-360 (2020). Article.

39. Pokrovskii, V. L. \& Uimin, G. V. Dynamics of vortex pairs in a two-dimensional magnetic material. JETP Lett. 41, 128 (1985). 
40. Papanicolaou, N. \& Spathis, P. N. Semitopological solitons in planar ferromagnets. Nonlinearity 12, 285-302 (1999). URL https:// doi .org/10 .1088/0951-7715/12/2/008.

41. Cooper, N. R. Solitary waves of planar ferromagnets and the breakdown of the spin-polarized quantum hall effect. Phys. Rev. Lett. 80, 4554-4557 (1998). URL https://link.aps.org/doi/10.1103/PhysRevLett.80.4554.

42. Huang, Y., Kang, W., Zhang, X., Zhou, Y. \& Zhao, W. Magnetic skyrmion-based synaptic devices. Nanotechnology 28, 08 LT02 (2017). URL http://stacks.iop.org/0957-4484/28/i=8/a=08LT02.

43. Fernández-Pacheco, A. et al. Three-dimensional nanomagnetism. Nature Communications $\mathbf{8}$, 15756 (2017).

\section{Methods}

Sample Fabrication $\mathrm{GdCo}_{2}$ micropillars of diameter $5 \mu \mathrm{m}$ were cut from a larger nugget of $\mathrm{GdCo}_{2}$ using a focused ion beam in combination with a micromanipulator, and mounted on top of OMNY tomography pins ${ }^{44}$.

The crystal structure of the $\mathrm{GdCo}_{2}$ micro-pillars was determined using microcrystallography measurements, performed at the X06DA beamline at the Swiss Light Source, Paul Scherrer Institute. An example diffraction pattern is given in Extended Data Figure 1, where one can observe that the Bragg peaks (right image) display a substructure, indicating the polycrystalline nature of the micropillar. 

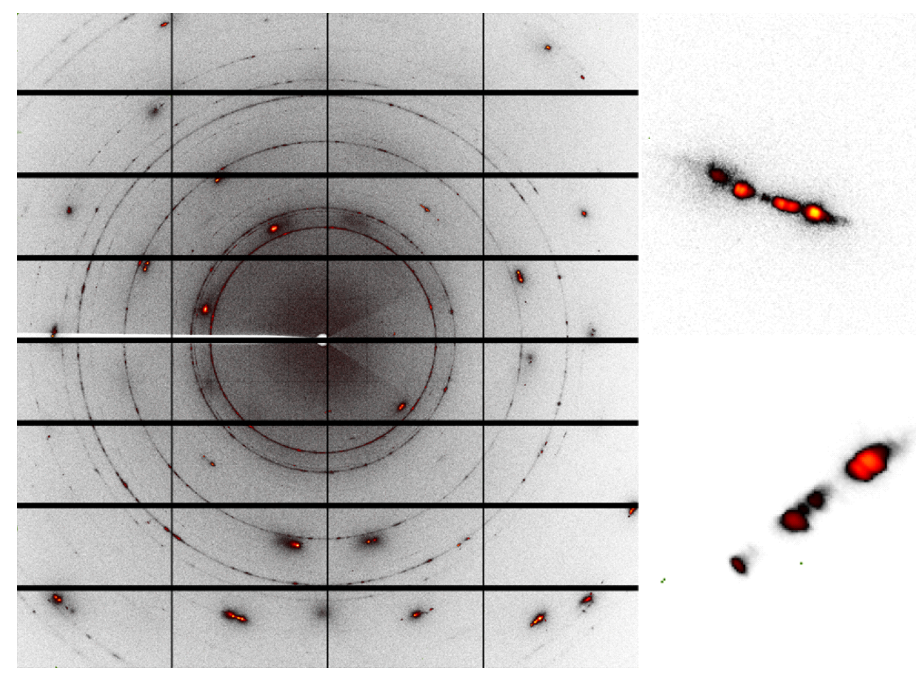

Extended Data Figure 1: A diffraction pattern from the $\mathrm{GdCo}_{2}$ pillar. The substructure of the Bragg peaks, magnified in the inset to the right, indicates the polycrystalline nature of the material.

X-ray ptychographic tomography Hard X-ray magnetic tomography was performed at the cSAXS beamline at the Swiss Light Source, Paul Scherrer Institut, using the flexible tomographic nano imaging (flOMNI) instrument ${ }^{45}$. Part of the data presented in this manuscript (the central vortex containing the Bloch point in Figure 2a,b) formed part of the dataset presented in Ref. 6. All other measurements and analysis are shown here for the first time here.

Two dimensional tomographic projections were measured with X-ray ptychography, a coherent diffractive imaging technique allowing access to the full complex transmission function of the sample ${ }^{46,47}$. For X-ray ptychography, an X-ray illumination of approximately $4 \mu \mathrm{m}$ was defined on the sample, and ptychography scans were performed by measuring diffraction patterns on a concentric grid of circles with a radial separation of $0.4 \mu \mathrm{m}$ for a field of view of $8 \times 7 \mu \mathrm{m}^{2}$ and $13 \times 9 \mu \mathrm{m}^{2}$ for the untilted and tilted sample orientations, respectively. The projections were recon- 
structed using 500 iterations of the difference map and 200 iterations of the maximum likelihood refinement using the cSAXS PtychoShelves package ${ }^{48}$.

To probe the magnetisation of the sample, X-rays tuned to the $\mathrm{Gd} L_{3}$ edge with a photon energy of $7.246 \mathrm{keV}$ were chosen to maximise the absorption XMCD signal ${ }^{23}$. Circularly polarised X-rays were produced by including a $500 \mu \mathrm{m}$-thick diamond phase plate upstream of the sample position ${ }^{49}$. The degree of circular polarisation achieved was greater than $99 \%$, and with an transmission of approximately $35 \%$.

The tomographic projections were aligned with high precision as described in Ref. 6.

Magnetic tomography When a single circular polarisation projection is measured, the component of the magnetisation parallel to the X-ray beam is probed due to the XMCD, along with the electronic structure of the sample. To probe all three components of the magnetisation, projections were measured around a rotation axis for two orientations of the sample ${ }^{6}$ Generally, the magnetic contrast of a projection is isolated from other contrast mechanisms by measuring the same projection using circular left and right polarised light, where the sign of the magnetic contrast is reversed, and taking the difference between the two images. Here, a single X-ray polarisation is used for all measurements and, in order to isolate the magnetic structure, projections with circularly left polarisation are measured at $\theta$ and $\theta+180^{\circ}$. Between these two angles, the magnetic contrast is reversed, which can be used to differentiate the magnetic contrast from the electronic contrast. Therefore, for the magnetic tomography measurements, circular left polarisation projections were measured through $360^{\circ}$ about the rotation axis, instead of through $180^{\circ}$, as in standard tomography. 
The magnetisation (which is a three-dimensional vector field) was reconstructed using a twostep gradient-based iterative reconstruction algorithm, described in Ref. ${ }^{50}$. The spatial resolution for each component of the magnetisation was estimated using Fourier Shell Correlation ${ }^{51}$, and a three-dimensional Hanning low-pass filter was used to remove high-frequency noise. The spatial resolution of the reconstructed magnetisation was found to be $97 \mathrm{~nm}, 125 \mathrm{~nm}$ and $127 \mathrm{~nm}$ in the $x-z, x-y$ and $y-z$ planes, respectively ${ }^{6}$.

The magnetic vorticity was calculated according to Equation 1. The magnetisation was normalised to obtain the unit vector, which was used to calculate the magnetic vorticity numerically in MATLAB. Specifically, the components of the vorticity vector were calculated numerically as follows:

$\Omega_{x}=2 m_{x}\left(\partial_{y} m_{y} \partial_{z} m_{z}-\partial_{z} m_{y} \partial_{y} m_{z}\right)+2 m_{y}\left(\partial_{y} m_{z} \partial_{z} m_{x}-\partial_{z} m_{z} \partial_{y} m_{x}\right)+2 m_{z}\left(\partial_{y} m_{x} \partial_{z} m_{y}-\partial_{z} m_{x} \partial_{y} m_{y}\right)$ $\Omega_{y}=2 m_{x}\left(\partial_{z} m_{y} \partial_{x} m_{z}-\partial_{x} m_{y} \partial_{z} m_{z}\right)+2 m_{y}\left(\partial_{z} m_{z} \partial_{x} m_{x}-\partial_{x} m_{z} \partial_{z} m_{x}\right)+2 m_{z}\left(\partial_{z} m_{x} \partial_{x} m_{y}-\partial_{x} m_{x} \partial_{z} m_{y}\right)$ $\Omega_{z}=2 m_{x}\left(\partial_{x} m_{y} \partial_{y} m_{z}-\partial_{y} m_{y} \partial_{x} m_{z}\right)+2 m_{y}\left(\partial_{x} m_{z} \partial_{y} m_{x}-\partial_{y} m_{z} \partial_{x} m_{x}\right)+2 m_{z}\left(\partial_{x} m_{x} \partial_{y} m_{y}-\partial_{y} m_{x} \partial_{x} m_{y}\right)$

where $m_{i}$ is the $i$ th component of the reduced magnetisation, and $\partial_{i}$ represents the partial derivative with respect to the $i$ th direction that were calculated numerically using the gradient function in MATLAB 2018a.

The three-dimensional visualisations of the magnetic vorticity and magnetisation were performed with Paraview ${ }^{52}$.

To consider the topology of the magnetisation in three dimensions, preimages corresponding 
to different directions are plotted within the pillar. The difference between the magnetisation vector and the $m_{x}=1$ direction is calculated using:

$$
\delta_{p x}=\left(\frac{m_{x}}{|\boldsymbol{m}|}-1\right)^{2}+\left(\frac{m_{y}}{|\boldsymbol{m}|}\right)^{2}+\left(\frac{m_{z}}{|\boldsymbol{m}|}\right)^{2}
$$

To plot the $m_{x}=1$ pre-image, for example, we plot an isosurface for $\delta_{p x}=0.01$. This results in a tube rather than a line, which is necessary due to the finite spatial resolution and signal-to-noise ratio of the measurement.

Field and thermal protocols A separate $\mathrm{GdCo}_{2}$ micropillar was used to investigate the effect of two different protocols, and the magnetic state was determined using magnetic tomography. The first protocol involved the application of a $7 \mathrm{~T}$ saturating field at room temperature. The second involved thermal annealing, (heating the micropillar to a temperature of $400 \mathrm{~K}$ close to the Curie temperature of the material), applying a $7 \mathrm{~T}$ field, and then reducing the temperature to room temperature, followed by a slow reduction of the applied magnetic field.

In the final states, a significant difference in both the presence of high vorticity structures, as well as the number of Bloch points present in the configuration, was observed. This can be seen in Figures 4 and Extended Data Figure 6, with the thermal annealing procedure resulting in a significant decrease in the average magnetic vorticity as well as in the number of Bloch points.

We note that, although the general magnetic structure is significantly different following the different protocols, and a large reduction in the average magnetic vorticity is observed following the annealing process, the main vortex that spans most of the height of the pillar occupies a similar 


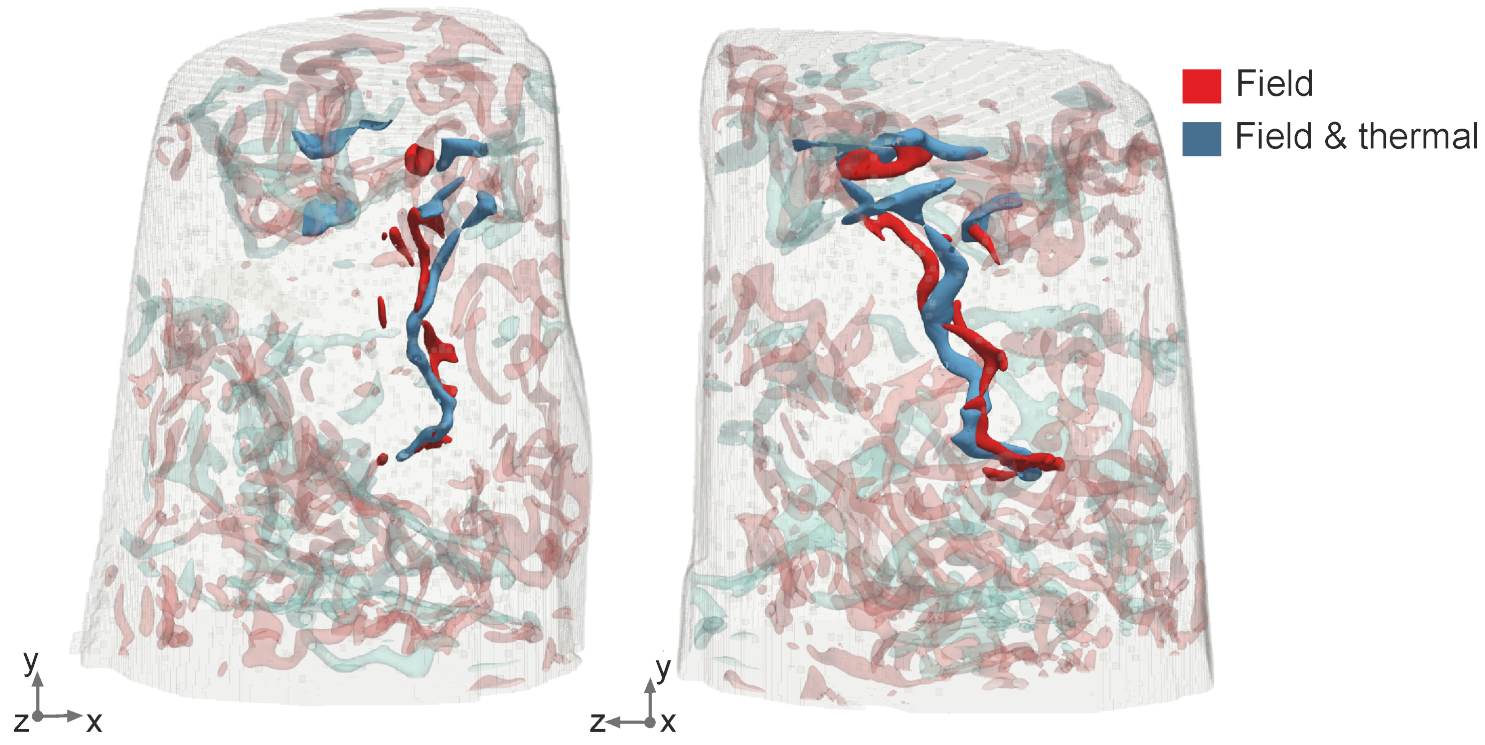

Extended Data Figure 2: Location of the central vortex following the two different protocols. The position of the central vortex core is plotted using red and blue isosurfaces for the remanent magnetic structure after (red) the application of a $7 \mathrm{~T}$ magnetic field, and (blue) after the application of the field cooling protocol. After both protocols, the vortex core returns to almost the same position.

position, within approx. $300 \mathrm{~nm}$ of the previous vortex, as can be seen in Extended Data Figure 2. Given that the vortex state is in principle the ground state of a cylindrical sample, the formation of the vortex core at nearby locations in a structure of this size is indicative of the presence of pinning centres that may be attributed to the polycrystalline nature of the material. The suppression of high vorticity structures, as well as magnetic vortex rings, following the thermal annealing protocol (see Extended Data Figure 6) indicates, however, that the pinning centres do not solely determine the stability of the structures, but rather may indirectly influence them through the pinning of neighbouring magnetic features. 
Analytical models To qualitatively interpret and understand the observed structures, we build a series of 2+1 dimensional models, which allow comparing the observed magnetization structures, preimages and the vorticity with the ones derived from modeled vortex loops with different magnetization structures. These models are similar to those used for description of hopfions in Ref.53. They are based on the subdivision of the magnetic material volume into thin slices, lying in the $x-y$ plane of a Cartesian coordinate system. The magnetisation in each slice can then be described by a complex function $w$ of a complex variable $u=x+\imath y$ by means of stereographic projection $\left\{m_{\mathrm{x}}+\imath m_{\mathrm{y}}, m_{\mathrm{z}}\right\}=\{2 w, 1-w \bar{w}\} /(1+w \bar{w})$, where the over-line denotes complex conjugation, so that $\bar{u}=x-\imath y, \imath=\sqrt{-1}$. Without loss of generality, any three-dimensional magnetisation distribution $\mathbf{m}(x, y, z)$ can be described by a function $w=w(u, \bar{u}, z)$, which depends on the complex coordinate $u$ within each slice and the extra-dimensional variable $z$, identifying the slice.

For realistic models, including at least the exchange and the magnetostatic interactions, no exact solutions for non-uniform $w(u, \bar{u}, z)$ are known. However, if the magnetostatic interaction is neglected and $w(u, \bar{u}, z)$ is assumed to be weakly dependent on $z$, two large families of exact solutions exist for $w(u, \bar{u}, z)$ at a fixed $z$. These are solitons ${ }^{20}$, which are meromorphic functions $w(u, \bar{u}, z)=f(u, z)$, and singular merons ${ }^{54}$, which are functions with $|w(u, \bar{u}, z)|=1$ or $w(u, \bar{u}, z)=f(u, z) /|f(u, z)|$. Zeros of $f(u, z)$ correspond to the centers of magnetic vortices (or hedgehog-like structures, if the magnetisation vectors are rotated by $\pi / 2$ in the $x-y$ plane). The poles correspond to the centers of the magnetic antivortices (or saddles). From the stereographic projection it follows that for solitons $m_{z}=1$ in the centers of the vortices and $m_{z}=-1$ in the centers of antivortices. 
An example of meromorphic functions are the rational functions of a complex argument (quotient of two polynomials). They allow direct expression of the vortex/antivortex pair annihilation as a cancellation of two identical monomials, whereas creation is a time-reversed process. The topological charge (or Skyrmion number) in each slice is a conserved quantity ${ }^{20}$ in the sense that it cannot be changed by a smooth singularity-free variation of the magnetisation distribution. For the slices in the $x-y$ plane the topological charge density is the $z$-component of the vorticity $\Omega_{z}$ and the total charge is the integral of this density over the whole slice. Creation and annihilation of the vortex-antivortex pairs within the soliton is always accompanied by a singularity.

A vortex ring can be understood as a process of creation, separation, convergence and annihilation of a vortex-antivortex pair as the variable $z$ advances through the successive slices ${ }^{5}$. Consider

$$
w_{\mathrm{BPr}}(u, \bar{u}, z)=f(u, z)=\imath \frac{u-p(z)}{u+p(z)}=\imath \frac{u-\sqrt{1-(z / 2)^{2}}}{u+\sqrt{1-(z / 2)^{2}}}
$$

for an (arbitrary) range $-2<z<2$, where the specific expression for $p(z)$ was chosen to make the vortex and antivortex cores extend along arcs, as in the experimental data. It describes the creation of a vortex-antivortex pair at $x=y=0$ and $z=2$, the vortex and antivortex moving apart (with the maximum distance between their centres equal to 2 at $z=0$ ), then approaching each other again, and annihilating at $z=-2$. We call this model the Belavin-Polyakov ring because each slice is a Belavin-Polyakov soliton, described by a meromorphic $w(u, \bar{u}, z)$. The corresponding schematic magnetisation, set of preimages and vorticity are shown in Extended Data Figure 3a. A similar preimage patterns connecting two Bloch points were indeed observed in our sample. However, the corresponding vorticity distributions are different. Indeed, instead of a 
single centrally-symmetric vorticity bundle we reconstruct a pair of bundles, corresponding to the vortex and antivortex centers. Clearly, the pure Belavin-Polyakov ring model can not reproduce this feature.

To 'unbundle' the vortex and antivortex, we can use the instanton model ${ }^{54}$ by writing:

$$
w_{\mathrm{i}}(u, \bar{u}, z)= \begin{cases}f(u, z) / c(z) & |f(u, z)| \leq c(z) \\ f(u, z) /|f(u, z)| & d(z)>|f(u, z)|>c(z) \\ f(u, z) / d(z) & |f(u, z)|>d(z)\end{cases}
$$

where $d(z)=1 / c(z)$, assuming the same size for the vortex and antivortex cores. Choosing $c(z)=1-q+q|z| / 2<1$ allows the control of the size of the vortex and antivortex cores (where $m_{z} \neq 0$ ) at the central plane $z=0$ via the parameter $q$. The magnetisation, preimages and vorticity for such an instanton ring with $q=3 / 4$ are shown in Extended Data Figure $3 \mathrm{~b}$. While they reproduce qualitatively both the vorticity distribution and the preimages, shown in figures $3 \mathrm{~b}$ and $3 \mathrm{~g}$, the structure of the Bloch points is different. Indeed, the instanton ring has two hedgehog-type Bloch points (in which the magnetisation directions are opposite), whereas the observed structure, shown in figure 3, contains two different types of Bloch points. Additionally, this model differs from the observation in figure 3 in that singularities are absent at the transition from the experimentally-observed vortex and antivortex pair to a uniformly-magnetized region. The Bloch points in figure 3 rather coincide with the polarisation reversal of vortex and antivortex cores as they propagate through the volume of the sample. In order to analytically describe this structure, we first need to build a model for a vortex ring. 


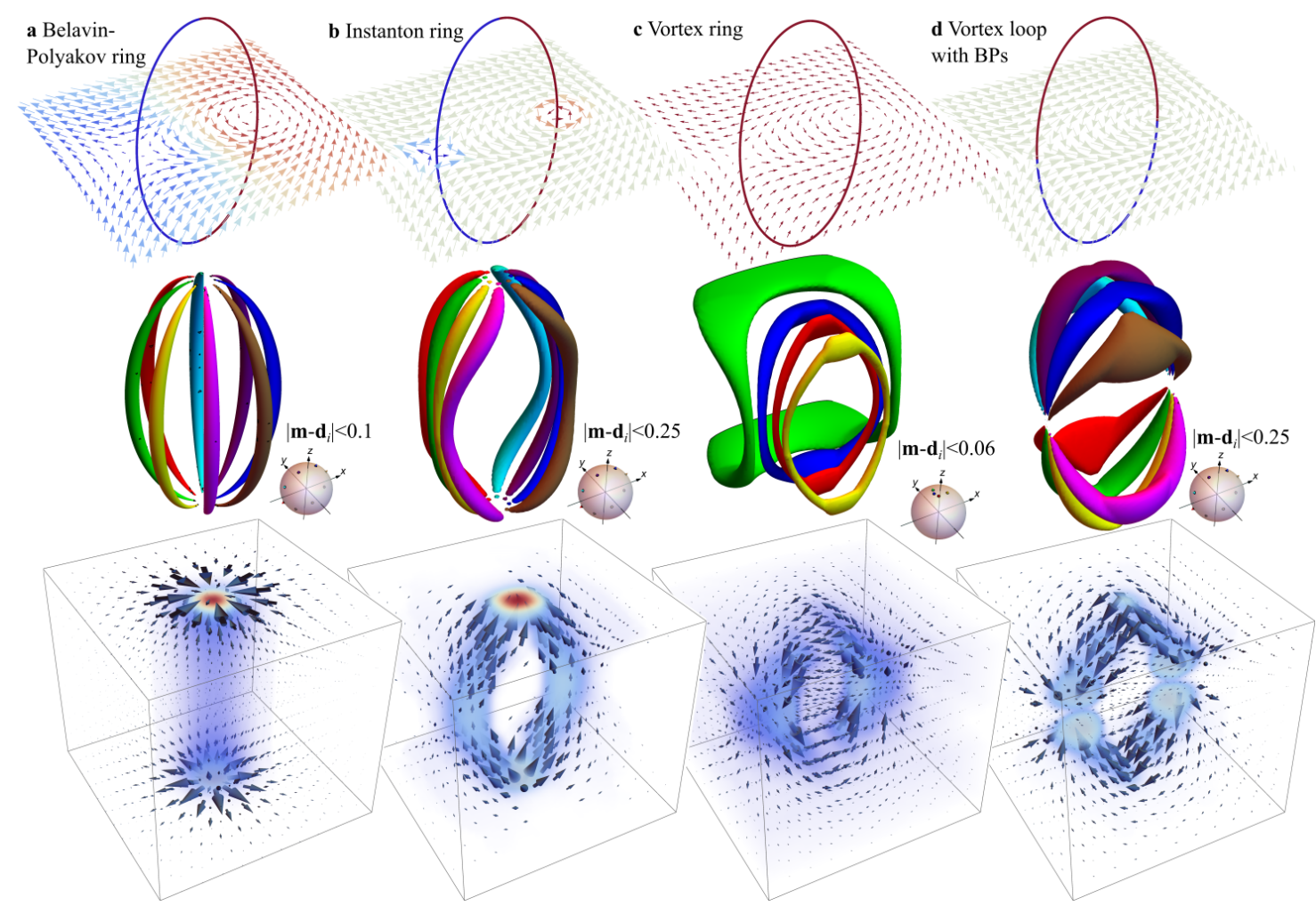

Extended Data Figure 3: Analytical models of vortex loops with different magnetisation structures. Top, middle and bottom rows: Magnetisation, pre-images and vorticity distribution for the different $2+1$ dimensional analytical models. The magnetisation plots (top row) only include the projection of the magnetisation onto the shown plane, while the rings correspond to the positions of the vortex and antivortex centers. The colour indicates the $m_{\mathrm{z}}$ component of the magnetisation. The preimages are shown as volumes where the magnetisation vectors deviate only slightly from certain directions $\mathbf{d}_{i}$, indicated by the color-coded arrows on each corresponding sphere. The opacity and color on the vorticity plots indicates the magnitude of local vorticity vectors. The structure in c) is comparable to the vortex rings in figure 2, while the structure in d) is comparable to that in figure 3. 
To describe a vortex-antivortex pair unbound by Bloch point singularities, the vortex and the antivortex must have identical polarisations (i.e. the same direction of $m_{z}$ within the core). In this case the topological charge in each slice is zero. Such a configuration can be obtained as a generalisation of (5)

$$
w_{\mathrm{r}}(u, \bar{u}, z)=A(z) \begin{cases}f(u, z) / c(z) & |f(u, z)| \leq c(z) \\ f(u, z) /|f(u, z)| & d(z)>|f(u, z)|>c(z) \\ d(z) / \overline{f(u, z)} & |f(u, z)|>d(z)\end{cases}
$$

where the modification to the last line reverses the polarisation of the antivortex. The factor $A(z)=$ $\left(1-z^{2} / 4\right) s$ ensures that, at $z= \pm 2$, the function $w_{\mathrm{r}}=0$, which corresponds to the uniform state. The parameter $s$ allows for the control of the degree of quasiuniformity: the smaller $s$ is, the less $m_{z}$ deviates from 1 . The magnetisation, preimages and vorticity for such a quasiuniform ring with $q=3 / 4$ and $s=1 / 4$ are shown in Extended Data Figure 3c. They are qualitatively analogous to the experimentally-observed vortex rings in figures $2 \mathrm{~b}$ and $2 \mathrm{~d}$.

Finally, we can extend the above model to a vortex ring in which the polarisation reverses along the vortex and the antivortex cores, in the presence of Bloch points. To describe this state, we note that with $s=1, c(z)=z^{2} / 4$, the magnetisation of the quasiuniform ring (6) at $z=0$ lies completely in the $x-y$ plane except for at the centres of the the vortex and antivortex, where its direction is undefined. Joining at the central plane two half-rings with opposite polarisations:

$$
w_{\mathrm{vls}}(u, \bar{u}, z)=A(z) \begin{cases}w_{\mathrm{r}}(u, \bar{u}, z) & z \leq 0 \\ 1 / \overline{w_{\mathrm{r}}(u, \bar{u}, z)} & z>0\end{cases}
$$


yields the model for the vortex loop with Bloch point singularities, shown in Extended Data Figure $3 \mathrm{~d}$. The structure corresponds well to the observations in figure 3, including the observed Bloch point types.

Note that despite the piecewise nature of the above functions, the resulting magnetisation vector fields are continuous (apart from at the singularities). While neither ansatz in the presented series is an exact solution of the corresponding micromagnetic problem (not even of its restricted exchange-only version), they provide a simple and easily interpretable model to understand the observed magnetisation distributions.

We now address the question of the size of the observed magnetisation structures. According to the Hobart-Derrick theorem, the exchange interaction alone cannot stabilize the solitons as the exchange energy does not display a minimum as function of the soliton size. However, the magnetostatic interaction, (which is outside of the scope of the Hobart-Derrick theorem) can, in principle, set the length scale of solitons. A complete answer to this question requires a sophisticated theoretical model, which still remains an open problem. Yet, a simple argument for stability of the observed bound states can be given in terms of other well-known magnetic textures such as a cross-tie wall as described below.

A single magnetic vortex, centered in a cylindrical nano-pillar, does not give rise to magnetic volume charges (which are proportional to the divergence of the magnetisation) and only generates surface charges (proportional to the magnetisation vector component, normal to the surface) in the region of the core at the surfaces of the pillar. The total energy (exchange plus surface mag- 
netostatic) of the magnetic vortex has a minimum when varying the vortex core size ${ }^{55}$. However, as the length of the pillar is increased to infinity, the equilibrium vortex core size diverges due to the diminishing role of the surfaces. In finite pillars, the vortex core has a barrel-like shape that is narrow at the top/bottom surfaces and wide in the middle of the pillar. These surface charges, however, do not explain the stability of the structures in the bulk of our pillar, which do not extend to the surfaces of the sample.

It is well known that, in thin films, vortices and antivortices can form bound states, such as in cross-tie walls ${ }^{56}$. A simple theoretical model for such a wall can be given directly in terms of the complex function $w$ of a (complex) variable $u^{57}$ :

$$
w_{\mathrm{c}-\mathrm{t}}(u, \bar{u}, z)=\imath \tan (u / s)
$$

where $s$ is the spatial scale (width) of the domain wall. The corresponding magnetisation structure has both volume and surface magnetic charges. The magnetostatic energy associated to these charges stabilizes the wall, yielding a certain equilibrium value of $s$ as a function of the film thickness $L$ and the exchange length $L_{\mathrm{EX}}=\sqrt{2 A /\left(\mu_{0} M_{\mathrm{S}}^{2}\right)}$, where $A$ is the exchange constant of the material. It should be noted, however, that, due to the presence of magnetic volume charges, the domain wall width for the model given by Equation (8) does not diverge as film thickness goes to infinity $L \rightarrow \infty$, but assumes a finite bulk limit

$$
s_{\infty}=8 \sqrt{\frac{3}{12-\pi^{2}}} L_{\mathrm{EX}}
$$

which can be directly computed using the magnetostatic function for the cross-tie wall ${ }^{57}$. For $\mathrm{GdCo}_{2}$ with an exchange length $L_{\mathrm{EX}} \simeq 20 \mathrm{~nm}$, the resulting value of $s_{\infty} \simeq 189 \mathrm{~nm}$, correspond- 
ing to the distances between vortex and antivortex centers of $s_{\infty} \pi / 2 \simeq 296 \mathrm{~nm}$, can serve as a ball-park theoretical estimate for the size of vortex rings.

Unlike a cross-tie domain wall, the magnetic vortex rings we observe are quasiuniform states and exist as a perturbation of a mostly uniform background. Because the magnetisation vector is included in both the exchange energy (squared gradients of components) and the magnetic volume charges density (product of divergences) via derivatives, a constant background is irrelevant and we can roughly assume that, in the quasiuniform state, only the spatial variation of the magnetisation vector is reduced, compared to the case of fully developed vortices and antivortices. For the quasiuniform cross-tie domain wall, this can be modeled by representing its total energy as

$$
E_{\mathrm{c}-\mathrm{t}} \propto c_{1} \frac{\left(L_{\mathrm{EX}} / L\right)^{2}}{s}+c_{2} F(s)
$$

where the case $c_{1}=c_{2}=1$ corresponds to the energy of the fully developed cross-tie wall ${ }^{57}$ and $F(s)$ is the magnetostatic function. The parameters $c_{1}$ and $c_{2}$ then account for the reduced variation of the magnetisation in the quasiuniform case, which has different effects on the exchange and magnetostatic energy terms. It is important to note that provided $c_{1}, c_{2} \neq 0$, this reduced variation does not destroy the energy minimum for $s$, but merely rescales the equilibrium wall width. This means that the quasiuniform bound state of vortices and antivortices can also be stable with respect to scaling, as for the cross-tie wall in a bulk magnet.

44. Holler, M. et al. Omny pina versatile sample holder for tomographic measurements at room and cryogenic temperatures. Review of Scientific Instruments 88, 113701 (2017). 


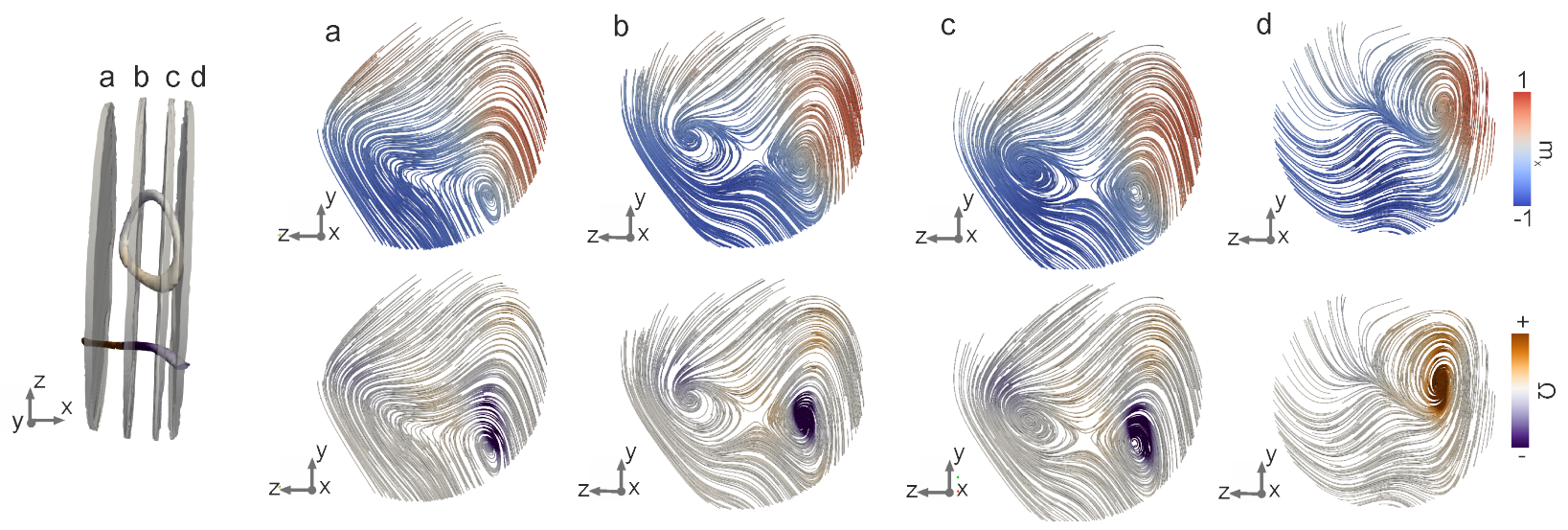

Extended Data Figure 4: Detailed overview of the vortex ring with circulating magnetic vorticity (presented in Figure2 of the main text), shown in successive slices through the loop. The magnetisation within each slice is represented by the streamlines The colourscale in the top row indicates the $\hat{x}$ component of the magnetisation, while the colour scale in the bottom row indicates the $\hat{x}$ component of the vorticity. The vorticity associated with the vortex structure extending throughout the pillar changes sign in slice $d$ due to the presence of a Bloch point, while the vortex-antivortex pair conserves its vorticity throughout. In slices $b$ and $c$, the magnetisation forms a structure similar to that of cross-tie walls, which dissolves as the pair unwinds, at slices $a$ and $d$, leaving the a single vortex. 

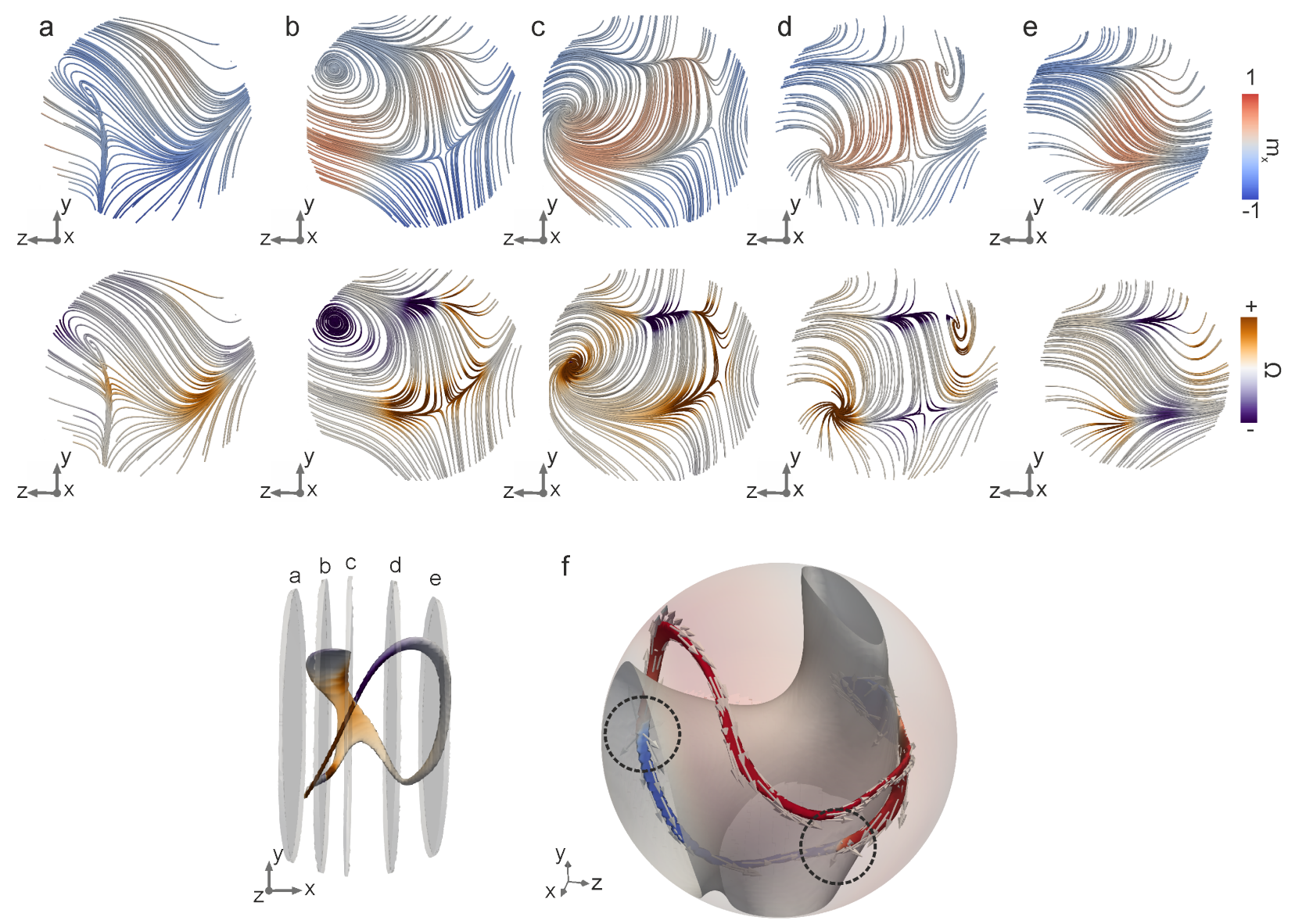

Extended Data Figure 5: Detailed overview of the magnetic state of the vortex loop containing Bloch points (presented in Figure3 of the main text), shown in successive slices through the loop. The magnetisation within each slice is represented by the streamlines. The colourscale in the top row indicates the $\hat{x}$ component of the magnetisation, while the colourscale in the bottom row indicates the $\hat{x}$ component of the vorticity. The vorticity along the vortex core reverses between slices $b$ and $c$, while the vorticity along the antivortex core reverses between slices $c$ and $d$. f) The white isosurface, plotted along with the vortex loop, corresponds to $m_{x}=0$ and separates regions of $m_{x}=+1$ and $m_{x}=-1$, thus highlighting the presence of a complicated domain wall structure. The Bloch points are located at the intersection of the loop with this isosurface (locations indicated by the dashed circles). 
a

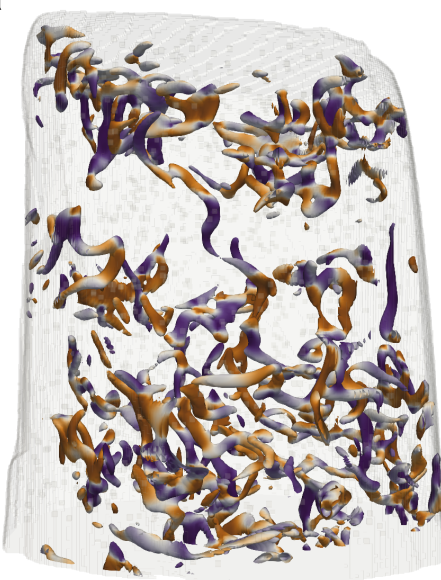

c

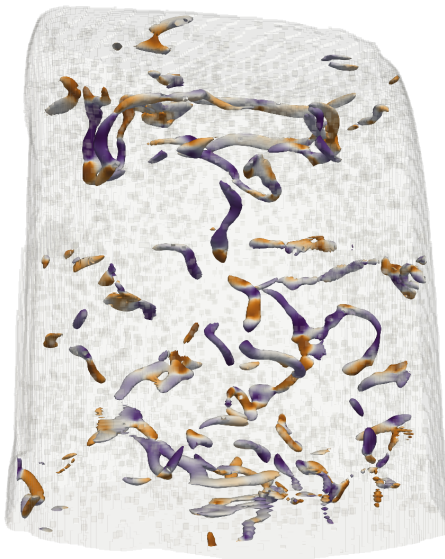

b
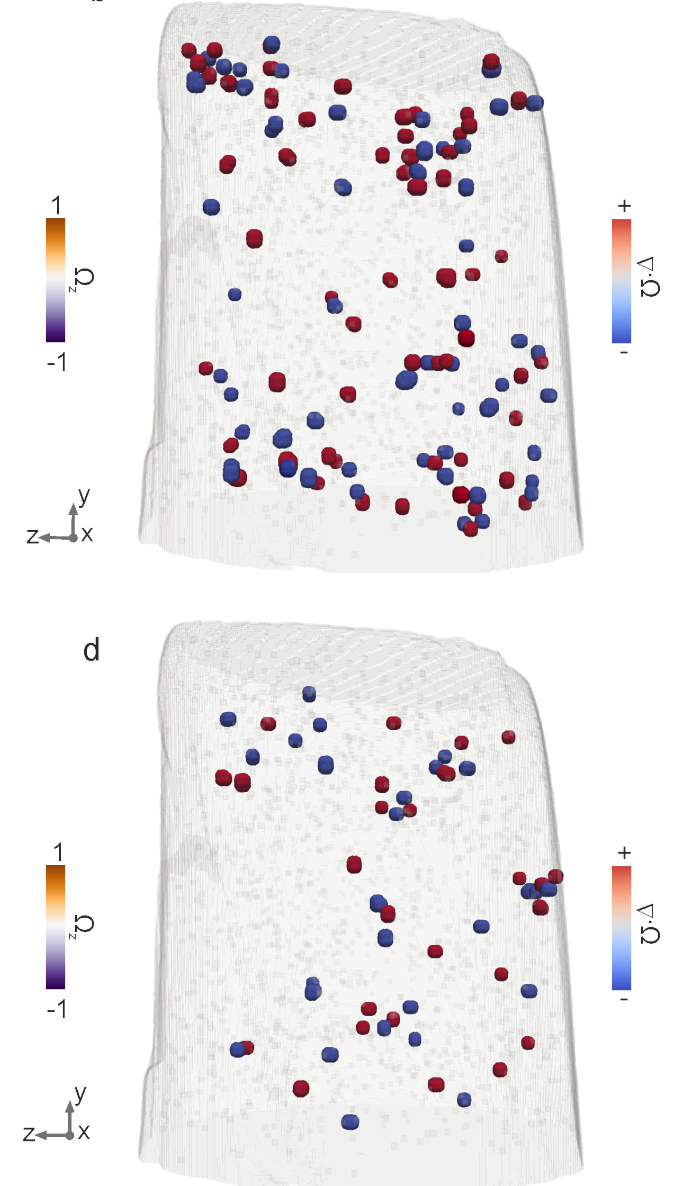

Extended Data Figure 6: Effect of different field and thermal protocols on the presence and distribution of regions of high magnetic vorticity, and magnetisation singularities. a) Vorticity distribution following the application of a $7 \mathrm{~T}$ saturating field and c) following saturation and field cooling. b) Regions of high divergence of the magnetic vorticity indicate the presence of Bloch points (red) and anti-Bloch points (blue)at remanence, following saturation. d) In the same way, singularities are identified after heating at $400 \mathrm{~K}$ and field cooling in a $7 \mathrm{~T}$ field. Noticeably fewer magnetic structures with high vorticity are present following the field cooling procedure. 
a

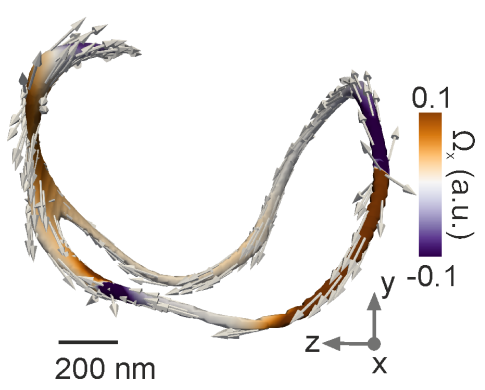

C

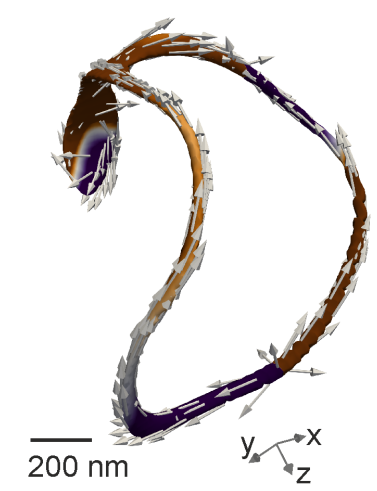

b

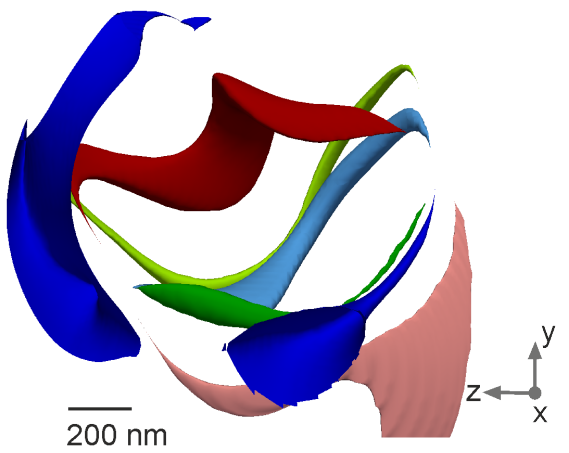

d

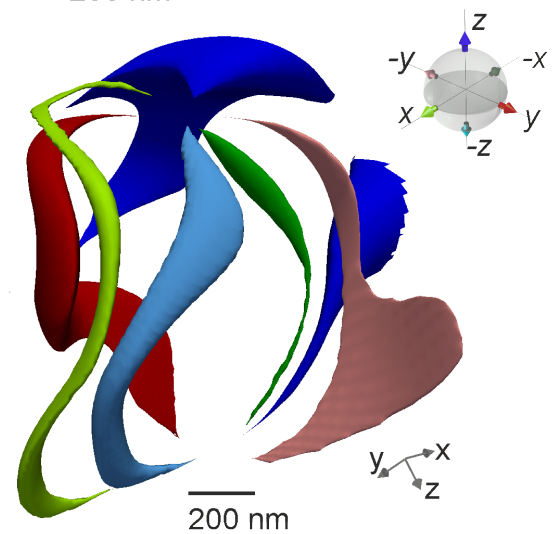

Extended Data Figure 7: The vortex loop containing magnetisation singularities (presented in Figure 3 in the main text ) seen from multiple directions. The vortex loop containing Bloch points is shown with the isosurface representing $m_{x}= \pm 1(\mathrm{a}, \mathrm{c})$ and pre-images $(\mathrm{b}, \mathrm{d})$. In a) and b), the vortex loop and its preimages have the same spatial orientation as in Figure $3 \mathrm{a}$ of the main text. In c) and d), the loop and preimages are presented with the same orientation as in Figure $3 \mathrm{~g}$. 
45. Holler, M. et al. High-resolution non-destructive three-dimensional imaging of integrated circuits. Nature 543, 402-406 (2017).

46. Pfeiffer, F. X-ray Ptychography. Nature Photonics 12, 9-17 (2017).

47. Rodenburg, J. M. et al. Hard-x-ray lensless imaging of extended objects. Phys. Rev. Lett. 98, 034801 (2007). URL https://link.aps.org/doi/10.1103/PhysRevLett.98.034801.

48. Wakonig, K. et al. PtychoShelves, a versatile high-level framework for high-performance analysis of ptychographic data. Journal of Applied Crystallography 53, 574586 (2020). URL https://doi.org/10.1107/S1600576720001776.

49. Scagnoli, V. et al. Linear polarization scans for resonant X-ray diffraction with a doublephase-plate configuration. Journal of Synchrotron Radiation 16, 778-787 (2009). URL https://doi.org/10.1107/s0909049509035006.

50. Donnelly, C. Hard X-ray Tomography of Three Dimensional Magnetic Structures. Ph. D. Thesis, ETH Zurich (2017).

51. van Heel, M. \& Schatz, M. Fourier shell correlation threshold criteria. JOURNAL OF STRUCTURAL BIOLOGY 151, 250-262 (2005).

52. Ahrens, J., Geveci, B. \& Law, C. ParaView: An End-User Tool for Large Data Visualization. Visualisation Handbook (Elsevier, 2005). 
53. Wilczek, F. \& Zee, A. Linking Numbers, Spin, and Statistics of Solitons. Phys. Rev. Lett. 51, 2250-2252 (1983).

54. Gross, D. J. Meron configurations in the two-dimensional O(3) $\sigma$-model. Nuclear Physics B 132, 439-456 (1978).

55. Usov, N. A. \& Peschany, S. E. Magnetization curling in a fine cylindrical particle. J. Magn. Magn. Mater. 118, L290-L294 (1993).

56. Huber, E. E., Jr., Smith, D. O. \& Goodenough, J. B. Domain-Wall Structure in Permalloy Films. J. Appl. Phys. 29, 294-295 (1958).

57. Metlov, K. L. Simple analytical description of the cross-tie domain wall structure. Appl. Phys. Lett. 79, 2609-2611 (2001).

\section{Contributions}

The study of topological magnetic features in three dimensions was conceived by S.G., C.D. and K.L.M., and originated from a larger project on three-dimensional magnetic systems conceived by L.J.H and J.R.. C.D., M.G.-S., S.G., V.S., M.H. and J.R. performed the experiments. Magnetometry measurements of the material were performed by N.S.B. and V.S.. C.D. performed the magnetic reconstruction with support from M.G.-S. and V.S.. C.D. analysed the data and N.R.C. conceived the calculation of the magnetic vorticity. C.D., K.L.M., N.R.C. and S.G. interpreted the magnetic configuration. K.L.M. developed the analytical model. C.D., K.L.M., N.R.C. and S.G. wrote the manuscript with contributions from all authors. 


\section{Acknowledgements}

X-ray magnetic tomography measurements were performed at the cSAXS beamline at the Swiss Light Source, Paul Scherrer Institut, Switzerland, and X-ray microcrystallography measurements at the X06DA beamline at the Swiss Light Source, Paul Scherrer Institut, Switzerland. The authors are grateful to Andrei Bogatyrëv for his careful reading of the manuscript and many valuable remarks, R. Cowburn for fruitful discussions, and V. Olieric for microcrystallography measurements. We thank R. M. Galera for providing and performing magnetic characterisations of the $\mathrm{GdCo}_{2}$ nugget, S. Stutz for the sample fabrication, and E. Müller from the Electron Microscopy Facility at PSI for the FIB-preparation of the pillar samples. C.D. is supported by the Leverhulme Trust (ECF-2018-016), the Isaac Newton Trust (18-08) and the LOral-UNESCO UK and Ireland Fellowship For Women In Science. S.G. was funded by the Swiss National Science Foundation, Spark Project Number 190736. K.L.M. acknowledges the support of the Russian Science Foundation under the project RSF 16-11-10349. N.R.C. was supported by EPSRC Grant EP/P034616/1 and by a Simons Investigator Award.

\section{Competing interests}

The authors declare no competing financial interests.

\section{Corresponding authors}

Correspondence to C.D., K.L.M. or S.G. 


\section{Data and Code Availability}

601 All data and codes will be made available on a repository following the publication of the manuscript. 